\title{
Role of Dectin-1 in the innate immune response of rat corneal epithelial cells to Aspergillus fumigatus
}

\author{
Qiang Xu, Guiqiu Zhao*, Jing Lin, Qian Wang, Liting Hu and Zhao Jiang
}

\begin{abstract}
Background: To observe Dectin-1 expression in fungal keratitis on rat models and to determine the role of Dectin-1 in innate immune response to Aspergillus fumigatus.

Methods: Wistar rats were randomly divided into control, fungal keratitis and pretreatment (pretreated with Laminarin) groups. Samples were used for conducting immunohistochemical staining and real-time PCR to observe expression of cytokines like CCL2, CCL3, CXCL1, CXCL2, IL-1ß, TNF-a, IL-6, IL-10.

Results: After fungal stimulations, all 7 inflammatory factors, except IL-10, increased with different levels. After $4 \mathrm{~h}$ of fungal stimulations, IL-1 $\beta, I L-6, C C L 2, C X C L 1$ and CXCL2 of pretreatment groups were significantly $(p<0.05)$ lower than fungal groups, while the other 3 cytokines had no significant changes. After $8 \mathrm{~h}$ of fungal stimulations, IL- 6 and CXCL1 of pretreatment groups were still significantly $(p<0.05)$ lower than fungal groups.

Discussion: With progress of fungus stimulation, expression of IL-1 $3, C X C L 1, C X C L 2, M C P-1$ gradually increased, whilepretreated with Laminarin to block Dectin-1, these expression decreased, indicating that Dectin-1 maypromote immune reaction through them. IL-10 decreased in fungal group because of itsimmunosuppressive effect at $4 \mathrm{~h}$, and it began to increase at $8 \mathrm{~h}$ to suppress Th1 inflammation response inorder to avoid excessive tissue damage.
\end{abstract}

Conclusion: Dectin-1 in early period of innate immune responses in rat fungal keratitis might work through IL-1 $\beta$, IL-6, CCL2, CXCL1, CXCL2 to recruit neutrophils and macrophages to participate anti-fungal immunity.

Keywords: Dectin-1, Innate immune, Corneal epithelium, Rat fungal keratitis, Laminarin

\section{Background}

Fungal keratitis (FK) is a serious eye disease which leads to blindness in some areas in China, especially for young adults. Therefore, it is very important to determine the pathogenesis of this disease and to find effective therapeutic targets. As the first line of defense in the immune response, innate immunity plays an important role in human defense of fungal infection. Pattern recognition receptors (PRRs) are key factors of innate immune response system, which could recognize pathogen-associated molecular patterns (PAMPs) and start a quick innate immune response, and then trigger persistent, specific adaptive immune response. Dectin- 1 , one of the $\beta$-glucan receptor expressed in dendritic cells and macrophages

* Correspondence: zhaoguiqiu_good@126.com

Affiliated Hospital of Qingdao University, 16th Jiangsu Road, Qingdao, Shandong Province, China plays a vital role in anti-fungal infection by recognizing fungi, recruiting immune cells, releasing inflammatory cytokines and starting an adaptive immune response.

Studies have shown that non-immune cells such as corneal epithelial cells and stromal cells may participate in innate immune responses through their own pattern recognition receptors. As the first line of defense, ocular corneal epithelium is not only a mechanical barrier to protect from the invasion of fungi, but also can identify the fungus through pattern recognition receptors [1]. Recent studies show that Dectin-1 plays an important role in the defense of the fungal infection [2]. Dectin-1 in the cornea can be unregulated after Fusarium and Aspergillus infection, while the Dectin-1 knockout rat is susceptible to Candida albicans and Aspergillus. When suffering from pulmonary Aspergillus fumigatus infection, Dectin-1 knockout rat had less lung inflammation 
response and more fungal load. Therefore, Dectin-1 expressed on corneal dendritic cells and macrophages play an important role in innate immune response by the early identification of Aspergillus fumigatus, recruitment of immune cells and release of inflammatory factors.

In this study, we pretreated rat corneal epithelium with Dectin-1 inhibitor laminarin (Laminarin), and then created fungal keratitis on rat models. The expressions of eight inflammatory factor (CCL2, CCL3, CXCL1, CXCL2, IL-1 $\beta$, TNF- $\alpha$, IL-6, IL-10) were monitored by immunohistochemistry and Real-time RT-PCR method in order to determine the role that Dectin-1 plays in early recruitment of inflammatory cells and to find its relationship with subsequent adaptive immunity response in fungal infections of the cornea.

\section{Methods}

Source of rat and fungal strains

All Wistar rats were approved by Qingdao medicine inspecting institute, Shandong province. They were healthy, ophthalmic diseases free, and treated in accordance with the guidelines provided in Scientific and Technological Commission of China for the Use of Animals for lab Research. Standard Aspergillus fumigatus strains (NO 3.0772) used in this study were purchased from China General Microbiological Culture Collection Center (CGMCC), and cultured according to standard procedure.

\section{Establishment of a rat model of Aspergillus keratitis}

Rat were randomly divided into control group $(n=6)$ and fungal keratitis group $(n=48)$. The fungal keratitis groups were divided into pretreatment group $(n=24)$ and fungi positive control group $(n=24)$. Pretreatment groups were pretreated with Laminarin eye dropping solution $(20 \mathrm{mg} / \mathrm{mL})$ for $2 \mathrm{~h}$, and fungal keratitis models were created at the same time with fungal keratitis group. The model was established and evaluated according to the criteria reported by $\mathrm{Li}$ et al. [3]. Corneal epithelium was collected at 4, 8, 16 and $24 \mathrm{~h}$ after the experimental model established for real-time PCR analysis. Eyeballs were collected at $24 \mathrm{~h}$ for immunohistochemistry.

\section{Real-time PCR}

Trizol Reagent was purchased from Invitrigen Prime Script RT reagent Kit With gDNA Eraser (Perfect Real Time) was purchased from TaKaRa. The house keeping gene GAPDH, primers and probes were purchased from TaKaRa. The sequences of the primers used were listed in Table 1.

To minimize the experimental error, total RNA was synchronously extracted from the corneal epithelium
Table 1 Sequence of GAPDH, CCL2, CCL3, CXCL1, CXCL2, IL-1ß, TNF- $a, I L-6$ and IL-10

\begin{tabular}{|c|c|c|c|}
\hline \multirow[b]{2}{*}{ GAPDH } & \multicolumn{2}{|c|}{ Sequence sequence $\left(5^{\prime} \rightarrow 3^{\prime}\right)$} & \multirow[t]{2}{*}{ Gene bank } \\
\hline & Sense & CCCCCAATGTATCCGTTGTG & \\
\hline & Antisense & GTAGCCCAGGATGCCCTITAGT & NM_017008 \\
\hline & Probe & TCTGACATGCCGCCTGGAGAAACC & \\
\hline \multirow[t]{3}{*}{ CCL2 } & Sense & ACCCATAAATCTGAAGCTA & \\
\hline & Antisense & GCATCACATTCCAAATCA & NM_031530.1 \\
\hline & Probe & TCCACAACCACCTCAAGCACT & \\
\hline \multirow[t]{3}{*}{ CCL3 } & Sense & GTCACATTTGTGTTTGTAG & \\
\hline & Antisense & CCTAGAATAATTGTCACCAA & NM_013025.2 \\
\hline & Probe & AAAGACCTCAGGGCACATTCC & \\
\hline \multirow[t]{3}{*}{ CXCL1 } & Sense & GGCTTCTGACAACACTAG & \\
\hline & Antisense & ACGAGATATTTAACGCCTAC & NM_030845.1 \\
\hline & Probe & CTGCACAATTGGAATTGAACGACCA & \\
\hline \multirow[t]{3}{*}{ CXCL2 } & Sense & GTGCCTAGATGTTGTTAC & \\
\hline & Antisense & CCTTCCAACTACATAAGTAA & NM_053647.1 \\
\hline & Probe & ATGCTGACTGAACACATTGAACATT & \\
\hline \multirow[t]{3}{*}{ IL-1 $1 \beta$} & Sense & CTTCGAGATGAACAACAA & \\
\hline & Antisense & CATGGAGAATACCACTTG & NM_031512.2 \\
\hline & Probe & ATGCCTCGTGCTGTCTGACC & \\
\hline \multirow[t]{3}{*}{ TNF-a } & Sense & CTGTCTACTGAACTTCGG & \\
\hline & Antisense & CATGGAACTGATGAGAGG & HQ201305.1 \\
\hline & Probe & TCCCAACAAGGAGGAGAAGTTCC & \\
\hline \multirow[t]{3}{*}{ IL-6 } & Sense & TCAGGAACAGCTATGAAG & \\
\hline & Antisense & AGTGGTATATACTGGTCTG & M26744.1 \\
\hline & Probe & CTTCCAGCCAGTTGCCTTCTTG & \\
\hline \multirow[t]{3}{*}{$\mid \mathrm{L}-10$} & Sense & GATCCAGAGATCTTAGCTA & \\
\hline & Antisense & CTGAGGTATCAGAGGTAA & L02926.1 \\
\hline & Probe & AACCTCGTTTGTACCTCTCTCCAA & \\
\hline
\end{tabular}

samples obtained at different observation points using Trizol reagent according to the manufacturer's protocol. Measurements of RNA concentration, reverse transcription and real-time quantitative PCR reactions were conducted in sequence.

\section{Immunocytochemistry}

Rabbit anti-rats CCL2, CCL3, CXCL1, CXCL2, IL-1 $\beta$, TNF- $\alpha$, IL-6, and IL-10 multi-clonal antibody, Histostain PLUS kit and DAB kit were purchased from Beijing Biosynthesis Biotechnology Co., Ltd.

Corneal paraffin sections, the thickness of $2 \mu \mathrm{m}$, were conventional dewaxed to water. S-P method was applied to stain every cytokine in corneal tissue. PBS buffer was used as negative control. Corneal tissue appeared brown 
particles for positive criteria. Field of vision was randomly selected and saved under 200 times zoom, the mean optical density of every cytokine staining was analyzed with VIDAS-21 (Japan SANYO) the computer color image analysis system.

\section{Statistical analysis}

All data were presented as mean \pm SD $(n=6)$. The data were analyzed with SPSS17.0 statistical package. Single factor analysis of variance was used to collectively comparison. The LSD tests were used to pairwise comparisons. The differences between groups were analyzed by $\mathrm{T}$ test. $P$ $<0.05$ was considered to be statistically significant.

\section{Results}

\section{Immunocytochemistry}

The changes of corneas on rat models after fungal stimulation in different time points were observed as showed in Fig. 1. Positive results of immunohistochemical staining can be seen in the cell. The greater inflammatory factors had deeper color in tissues. Results demonstrated that eight inflammatory factors were mainly expressed in cytoplasm in the corneal epithelium and shallow stroma. The fungal groups and pretreatment groups showed clear pictures by comparing with normal rat groups, while there were no obvious difference between fungal groups and pretreatment groups (Fig. 2).

\section{Real-time RT-PCR}

As shown in Figs. 3 and 4, expression of eight inflammatory factors mRNA was detected in every blank (normal rat) group. After $4 \mathrm{~h}$ of fungus stimulation, expression of IL-1 $\beta$, IL-6, CCL2, CXCL1, CXCL2 increased and had significant difference $(p<0.05)$. After $8 \mathrm{~h}$ of fungus stimulation, expression of TNF- $\alpha$ and CCL3 increased more and had significant difference $(p<0.05)$. Expression of IL-10 was lower in $4 \mathrm{~h}$ fungus groups than control groups, and at $8 \mathrm{~h}$ fungus groups it increased and was statistically significant compared with control groups $(p<0.05)$. IL-1 $\beta$, TNF- $\alpha$ and CCL3 reached maximum at $16 \mathrm{~h}$, and then decreased from $24 \mathrm{~h}$. CXCL1 increased from $4 \mathrm{~h}$ to $16 \mathrm{~h}$, and then decreased. IL-6 reached peak at $8 \mathrm{~h}$, then gradually decreased, while expression of CCL2 and CXCL2 continued to increase for all the time.

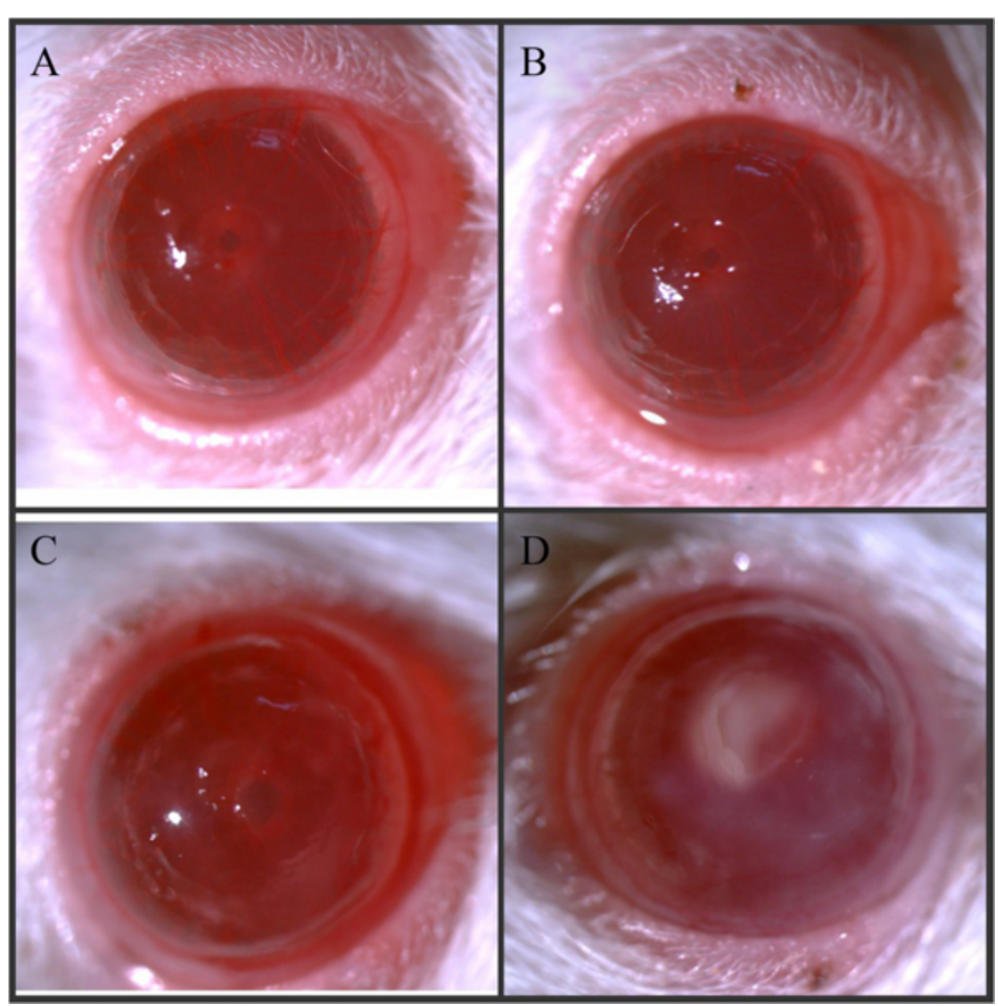

Fig. 1 Morphology of fungal infections models. a $4 \mathrm{~h}$ after fungal infection $\mathbf{b} 8 \mathrm{~h}$ after fungal infection $\mathbf{c} 16 \mathrm{~h}$ after fungal infection $\mathbf{d} 24 \mathrm{~h}$ after fungal infection 


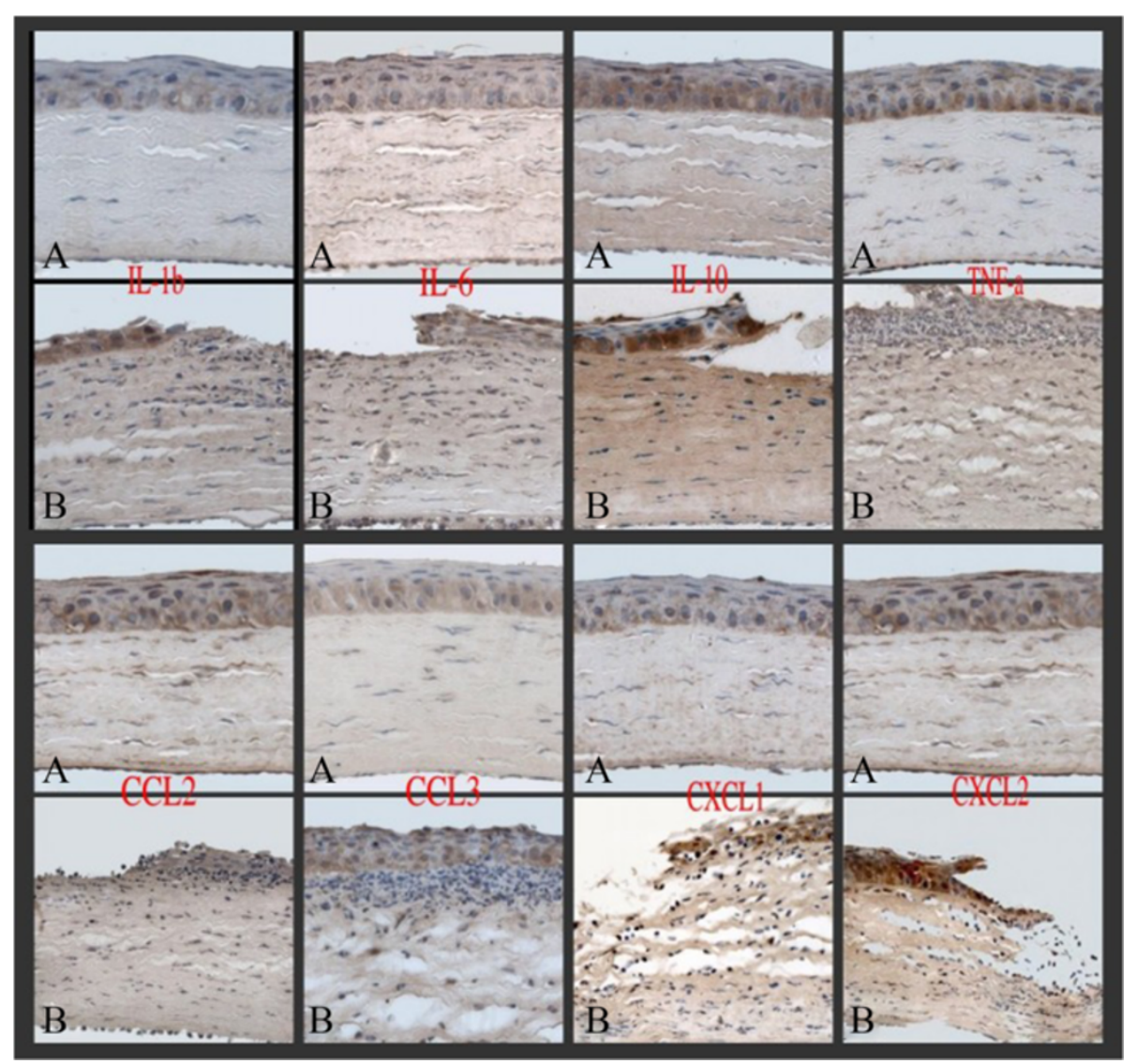

Fig. 2 Immunocytochemistry results immunohistochemical staining $\times 400$. a normal rat group $\mathbf{b}$ fungal group. The eight inflammatory factors were mainly expressed in cytoplasm in the corneal epithelium and shallow stroma. The fungal groups and pretreatment groups showed clear pictures by comparing with normal rat groups, while there were no obvious difference between fungal groups and pretreatment groups

\section{Discussion}

Laminarin, 1,3- $\beta$-glucan extracted from a Laminoriadigitata, can specifically bind Dectin-1. It is considered as Dectin-1 specific blocker. The study indicated that Laminarin connected with ovalbumin OVA could be specially recognized by Dectin-1 receptor expressed on dendritic cells and macrophage. $\mathrm{CD}^{+} \mathrm{T}$ cell responses could be induced through cross-presentation, leading to secretion of inflammatory factors by dendritic cells, which can verify that Laminarin could act as Dectin-1 specific blocker in the animal models [4].

Dectin-1 can recognize particulate and soluble $\beta$ glucans from fungi, bacteria, and plants, and is the primary receptor for these carbohydrates on leukocytes, through which it can induce the innate immune reaction and eradicate pathogens. Dectin-1 is expressed predominantly by myeloid cells in many tissues, with highest levels of expression on inflammatory cells and cells at portals of pathogen entry, such as alveolar macrophages [5]. In addition to macrophages, Dectin-1 is expressed on monocytes, neutrophils, and most subsets of dendritic cells as well as on subpopulations of $\mathrm{T}$ cells. Ocular Dectin-1 is mainly expressed in the corneal epithelial cells, macrophages and dendritic cells [6].

It was found that the susceptibility to Candida alricans in Dectin- $1^{-/-}$rat was declined with low level of inflammatory reaction and decreased fungal exterminating ability [7]. Many studies showed that Dectin-1 is able to induce ligand uptake by endocytosis and phagocytosis, the respiratory burst, the production of arachidonic acid metabolites, and the induction of numerous cytokines and chemokines. Spleen Tyrosine Kinase (Syk) is a central kinase and mediates most of the functions of Dectin-1 and this signaling pathways plays a critical role [8]. Signaling downstream from Syk involves the novel adaptor CARD9, and activation of mitogen-activated protein (MAP) kinases, nuclear factor of activated $\mathrm{T}$ cells (NFAT) and nuclear factor kappa B (NF-kB) [9-11] which finally results in expression of downstream factors like, including TNF, CXCL2, IL-23, IL-6, IL-10, IL-2, and IL-12 [12]. 


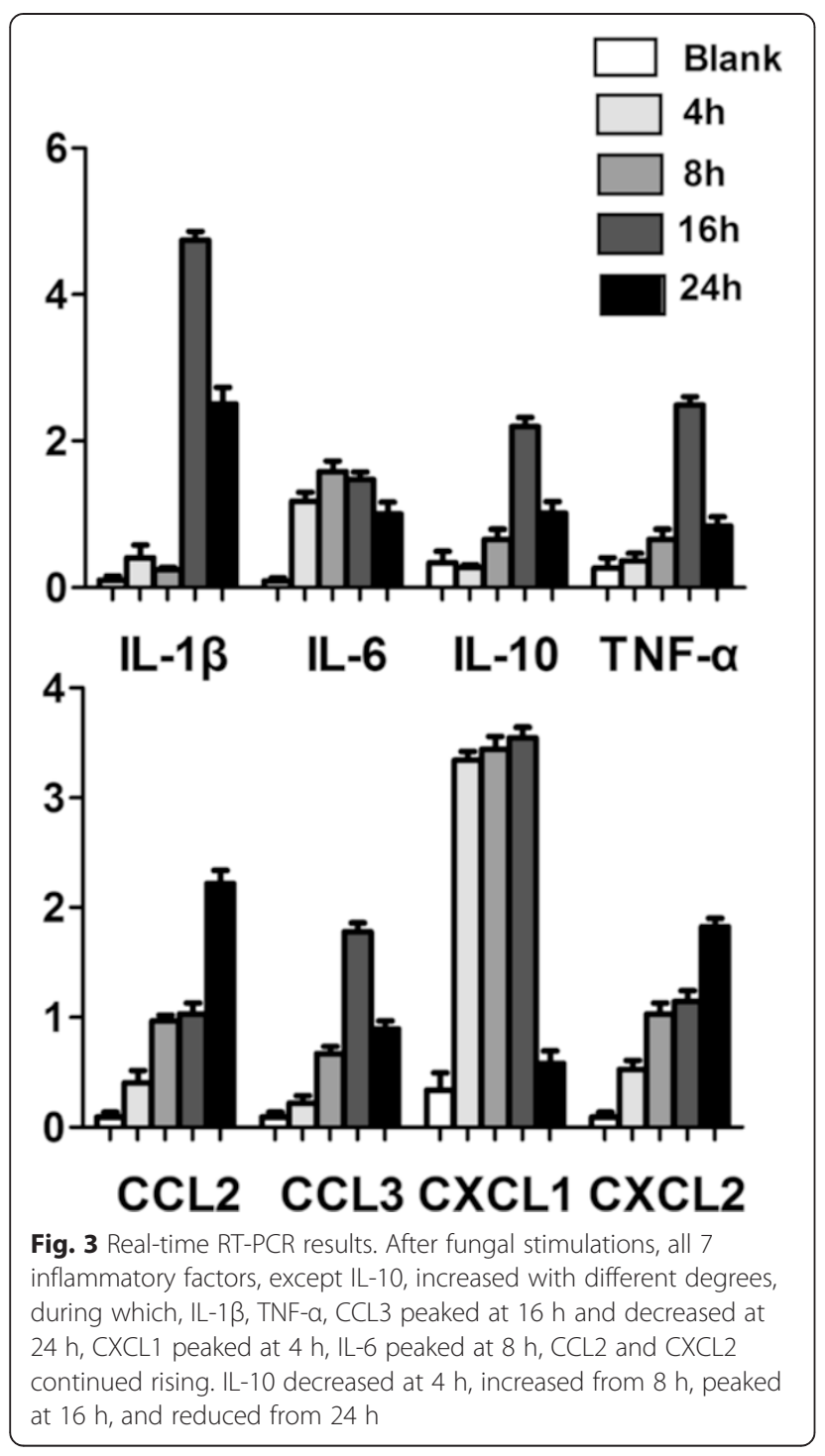

Studies have proved that Dectin-1 gene and protein can express in normal human corneal epithelium, and after Aspergillus fumigatus stimulation, synthesis of Dectin-1 was increased, suggesting that Dectin-1 could be activated in fungal keratitis. Leal et al. [2] has established a Dectin-1 deficient rat fungal keratitis models. They found that cell infiltration and fungi scavenging were significantly reduced compared with the control group, with decreased production of IL-1 $\beta$ and CXCL1. Therefore, it was thought that dectin-l had an important role in Aspergillus fungal keratitis, cytokine production, neutrophil and monocyte recruitment to the corneal stroma. The fungal killing is dependent on the presence of macrophages and dendritic cells, and on expression of Dectin-1.

Our data showed that IL-1 $\beta$ expression level was very low in the control group, and there was not significant increase in corneal epithelium in fungal groups at $4 \mathrm{~h}$, but its expression was significantly increased after $8 \mathrm{~h}$. We assumed that IL-1 $\beta$ was mainly produced by macrophages and monocytes, while raising these cells took some time after corneal fungal infection. We also found that expression of IL-1 $\beta$ lagged compared with monocyte chemoattractant CXCL1 and CXCL2. Pretreated with Laminarin to block Dectin-1 in corneal epithelium, IL-1 $\beta$ expression decreased significantly at $4 \mathrm{~h}$ after fungal stimulation.

The main biological activity of IL-10 is immunosuppressive, which can inhibit activation and effector function of $\mathrm{T}$ cell and monocyte-macrophage cells. Our study showed that IL-10 higher expression in blank group, and it decreased in fungal group at $4 \mathrm{~h}$. As a Th2-type cytokine, its expression in normal corneal epithelium could inhibit activation of immune cells to prevent immune response leading to tissue damage, and after fungal infections, innate immunity of the body is firstly activated, when the site of infection requires a lot of inflammatory cell, expression of IL-10 decreases to let its immunosuppressive effect down to help killing the fungus early. With progress of infection, immune response was constantly enhanced and expression of IL-10 began to increase at $8 \mathrm{~h}$ to suppress Th1 inflammation response in order to avoid excessive tissue damage. Pretreated with Laminarin could block Dectin-1 in corneal epithelium, thus IL-10 expression did not change after fungal stimulation.

Chemokines, mainly composed of macrophages, immune cells and non-immune cells, are involved in inflammatory process by leukocyte chemotactic and chemokinesis. Our study showed that with fungal stimulating, corneal epithelium expression of MIP-2 gradually increased, and pretreated with Laminarin to block Dectin-1 in corneal epithelium, expression of CXCL1 and CXCL2 decreased in $4 \mathrm{~h}$ of fungal stimulation, while CXCL1 was still less in pretreatment group than fungal group after $8 \mathrm{~h}$, and the difference was statistically significant, indicating that Dectin-1 may have neutrophil chemotaxis through both CXCL1 and CXCL2.

Monocyte chemotactic protein MCP-1, also known as CCL2, is major monocyte chemotaxis factor in inflammation. It can recruit monocytes, memory $\mathrm{T}$ cells and dendritic cells to the site of injury, infection and inflammation, then initiate factor of inflammatory cytokine network. Our results showed that with progress of fungus stimulation, expression of MCP-1 in corneal epithelium gradually increased, and pretreated with Laminarin to block Dectin-1 in corneal epithelium, expression of MCP-1 decreased at $4 \mathrm{~h}$, which was statistically significant, indicating that Dectin-1 may raise monocyte through MCP-1. 

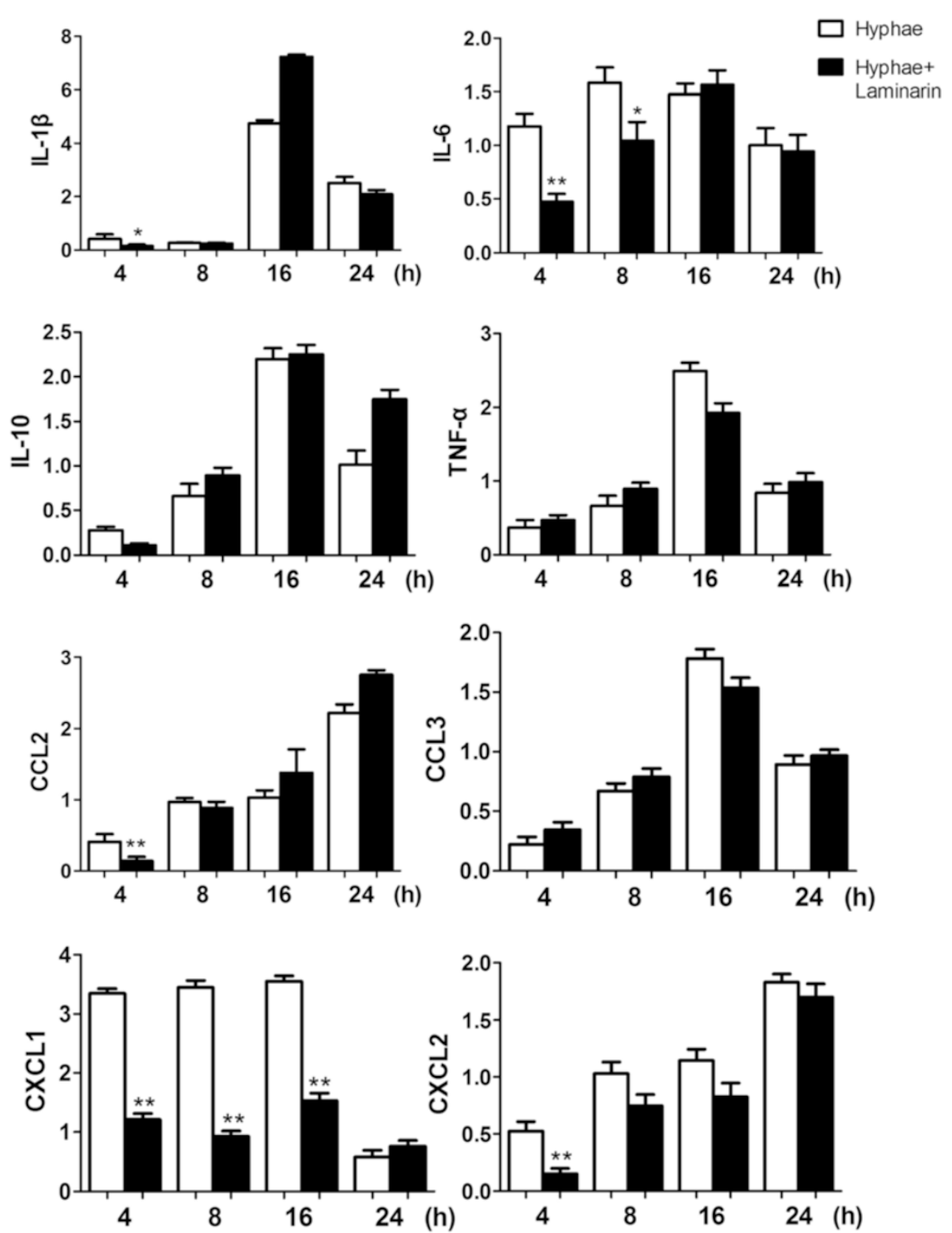

Fig. 4 Real-time RT-PCR results. After $4 \mathrm{~h}$ of fungal stimulations, IL-1 $\beta, I L-6, C C L 2, C X C L 1$ and CXCL2 of pretreatment groups were lower than fungus groups and was statistically significant $(p<0.05)$, while the other 3 factors with no statistically significant. After 8 h of fungal stimulations, IL-6 and CXCL1 of pretreatment groups were still lower than fungus groups and was statistically significant $(p<0.05)$

\section{Conclusions}

Our experiment confirmed that Dectin-1 in early period of innate immune in rat fungal keratitis can work through IL-1 $\beta$, IL-6, CCL2, CXCL1, CXCL2 to recruit neutrophils and macrophages to participate anti-fungal immunity. Cytokine production, neutrophil and monocyte recruitment and killing fungi are dependent on presence of macrophage and dendritic cells and expression of $\beta$-glucan receptor Dectin-1. Dectin-1, as specific innate immunity molecule modulators in development of this disease, would become targets for antifungal therapy.

\section{Competing interests}

There were no financial conflict interests and non-financial competing interests that may cause them embarrassment between all the authors.

\section{Authors' contributions}

$X Q$ carried out all this trials and drafted the manuscript. $\sqcup$ participated in the sequence alignment. WQ and HL participated in the design of the study and performed the statistical analysis. JN and JZ helped keeping animals. ZG conceived of the study and participated in its design and coordination and helped to draft the manuscript. All authors read and approved the final manuscript.

Authors' information Not applicable. 
Availability of data and materials

Not applicable.

\section{Acknowledgments}

I do not have someone to acknowledge.

\section{Funding}

This article was from the National Natural Science Foundation of China: The role and mechanism of innate immune mediated by Dectin-1 in fungal keratitis (NO:81170825).

This article was supported by National Natural Science Foundation of China (No.81170825:Role of Dectin-1in the innate immune inflammation of Fungal Keratitis; No.81470609:Mechanism of LOX-1 in anti-fungalinnate immune of fungal keratitis); Shandong province natural science foundation (No. ZR2013HQ007: Role of Interlein33/ST2 signaling in the immune inflammation of Fungal Keratitis; No. ZR2012HZ001: Theinfluence of AQP1 on a Crystal protein and the study of targeted therapy in the pathogenesis of senilecataract ); the Specialized Research Fund for the Doctoral Program of Higher Education, 2012(No.20123706110003: Role of Mincle in the innate immune inflammation of Fungal Keratitis).

Received: 24 November 2014 Accepted: 10 September 2015

Published online: 01 October 2015

\section{References}

1. Zhao J, Wu X-y, Fu-Shin X, Yu. Activation of Toll-like receptors 2 and 4 in Aspergillus fumigatus keratitis. Innate Immunity. 2009;15:155-68.

2. Leal Jr SM, Cowden S, Hsia Y-C, Ghannoum MA, Momany M, Pearlman E. Distinct Roles for Dectin-1 and TLR4 in the Pathogenesis of Aspergillus fumigatus Keratitis. PLoS Pathogens. 2010;6:e1000976.

3. Li N, Che CY, Hu LT, Lin J, Wang Q, Zhao GQ. Effects of COX-2 inhibitor NS-398 on IL-10 expression in rat fungal keratitis. Int J Ophthalmol. 2011;4(2):116-20.

4. Xie J, Guo L, Ruan Y, Zhu H, Wang L, Zhou L, et al. Laminarin-mediated targeting to Dectin-1 enhances antigen-specific immune responses. Biochemical and Biophysical Research Communications. 2010;391:958-62.

5. Reid DM, Montoya M, Taylor PR, Borrow P, Gordon S, Brown GD, et al. Expression of the \{beta\}-glucan receptor, Dectin-1, on murine leukocytes in situ correlates with its function in pathogen recognition and reveals potential roles in leukocyte interactions. J Leukoc Biol. 2004;76:86-94.

6. Li N, Zhao GQ. Mechanism of the immune response to keratomycosis. Chin J Ophthalmol. 2011;47:378-81.

7. Taylor PR, Tsoni SV, Willment JA, Dennehy KM, Rosas M, Findon H, et al. Dectin-1 is required for beta-glucan recognition and control of fungal infection. J Nat Immunol. 2007;8(1):31-8.

8. Mohlopheni J M,Ann M K, Gordon D B. Dectin-1: a role in antifungal defense and consequences of genetic polymorphisms in humans. Mammalian Genome. 2011;22(1-2):55-65

9. Goodridge HS, Simmons RM, Underhill DM. Dectin-1 stimulation by Candida albicans yeast or zymosan triggers NFAT activation in macrophages and dendritic cells. J Immunol. 2007;178:3107-15.

10. Gross O, Gewies A, Finger K, Schäfer M, Sparwasser T, Peschel C, et al. Card9 controls a non-TLR signalling pathway for innate anti-fungal immunity. Nature. 2006;442:651-6.

11. Slack EC, Robinson MJ, Hernanz-Falcon P, Brown GD, Williams DL, Schweighoffer $\mathrm{E}$, et al. Syk-dependent ERK activation regulates IL-2 and IL-10 production by DC stimulated with zymosan. Eur J Immunol. 2007;37:1600-12.

12. Gantner BN, Simmons RM, Canavera SJ, Akira S, Underhill DM. Collaborative induction of inflammatory responses by Dectin-1 and Toll-like receptor 2. J Exp Med. 2003;197(9):1107-17.

\section{Submit your next manuscript to BioMed Central and take full advantage of:}

- Convenient online submission

- Thorough peer review

- No space constraints or color figure charges

- Immediate publication on acceptance

- Inclusion in PubMed, CAS, Scopus and Google Scholar

- Research which is freely available for redistribution

Submit your manuscript at www.biomedcentral.com/submit 